\title{
Estimation of Approximation with Jacobi Weights by Multivariate Baskakov Operator
}

\author{
Jianjun Wang, Haifeng Guo, and Jia Jing \\ School of Mathematics and Statistics, Southwest University, Chongqing 400715, China \\ Correspondence should be addressed to Jianjun Wang; wjj@swu.edu.cn
}

Received 7 May 2013; Accepted 14 July 2013

Academic Editor: Yongsheng S. Han

Copyright (C) 2013 Jianjun Wang et al. This is an open access article distributed under the Creative Commons Attribution License, which permits unrestricted use, distribution, and reproduction in any medium, provided the original work is properly cited.

\begin{abstract}
We first give the unboundedness of multivariate Baskakov operators with the normal weighted norm. By introducing new norms, using the multivariate decomposition technique and the modulus of smoothness with Jacobi weight, the upper bound estimation of multivariate Baskakov operators is obtained. The obtained results not only generalize the corresponding ones for multivariate Baskakov operators without weights, but also give the approximation accuracy with the Jacobi weights approximation.
\end{abstract}

\section{Introduction and Main Results}

Let $C_{B}[0,+\infty)$ be the set of bounded continuous functions on $[0,+\infty)$; then the unvariate Baskakov operator is defined by

$$
V_{n, 1}(f, x)=\sum_{k=0}^{\infty} v_{n, k}(x) f\left(\frac{k}{n}\right)
$$

where

$$
v_{n, k}(x)=\left(\begin{array}{c}
n+k-1 \\
k
\end{array}\right) x^{k}(1+x)^{-n-k}, \quad x \in[0, \infty] .
$$

There are many papers to study the univariate Baskakovtype operator (see [1-4]). In [5], the authors discussed the convergence property of Baskakov operator's approximation with weights by introducing weighted smoothness modulus and obtained the characteristics of approximation. And in [6], using the weighted smoothness modulus, the close connection between the derivative of Baskakov operator and the smoothness of function approximated which has been investigated, the upper bound estimation has been established with the Jacobi weight; that is, for any $f \in$ $C_{\omega}, 0 \leq \lambda \leq 1$,

$$
\left|\omega(x)\left(V_{n, 1}(f, x)-f(x)\right)\right| \leq C \omega_{\varphi^{\lambda}}^{2}\left(f ; n^{-1 / 2} \varphi^{1-\lambda}(x)\right)_{\omega},
$$

where $C_{\omega}=\left\{f \mid f \in C[0, \infty], \omega f \in L_{\infty}[0,+\infty]\right\}$. In this paper, the letter $C$, appearing in various formulas, denotes a positive constant independent of $n, x$, and $f$. Its value may be different at different occurrences, even within the same formula.

Let $T \in R^{d}(d \in N)$, let $T=T_{d}=\mathbf{x}=\left\{\left(x_{1}, x_{2}, \cdot, x_{d}\right)\right.$, and let $\left.0 \leq x_{i}<\infty, 0 \leq i \leq d\right\}$,

$$
\begin{aligned}
& \mathbf{x}=\left(x_{1}, x_{2} \cdots x_{d},\right), \quad \mathbf{k}=\left(k_{1}, k_{2}, \ldots, k_{d}\right), \\
& \mathbf{x}^{\mathbf{k}}=x_{1}^{k_{1}} x_{2}^{k_{2}} \cdots x_{d}^{k_{d}}, \\
& |\mathbf{k}|=k_{1}+k_{2}+\cdots+k_{d}|\mathbf{x}|=x_{1}+x_{2}+\cdots+x_{d}, \\
& \left(\begin{array}{l}
n \\
\mathbf{k}
\end{array}\right)=\frac{n !}{\mathbf{k} !(n-|\mathbf{k}|)}, \quad \sum_{\mathbf{k}=0}^{\infty}=\sum_{k_{1}=0}^{\infty} \sum_{k_{2}=0}^{\infty} \cdots \sum_{k_{d}=0}^{\infty}, \\
& C_{\varphi}^{r}(T)=\left\{f \in C_{B}(T): f \in C_{B}^{r}(T),\right. \\
& \text { and } \left.\varphi_{i}^{r} D_{i}^{r} f \in C_{B}(T), 1 \leq i \leq d\right\},
\end{aligned}
$$

where $C_{B}(T)$ represents the set of bounded continuous functions in $T$. The norm defined on $T$ is $\|f\|_{\infty}=\max _{x \in T}|f(x)|$.

Using the previous notations, the multivariate Baskakov operator is defined by

$$
V_{n, d}(f ; \mathbf{x})=\sum_{\mathbf{k}=0}^{\infty} v_{n, \mathbf{k}}(\mathbf{x}) f\left(\frac{\mathbf{k}}{n}\right),
$$


where $v_{n, \mathbf{k}}(\mathbf{x})=\left(\begin{array}{l}n+|\mathbf{k}|-1 \\ \mathbf{k}\end{array}\right) \mathbf{x}^{\mathbf{k}}(1+\mathbf{x})^{-n-|\mathbf{k}|}$. In [7], the authors gave the equivalent relation between $K$-functional and modulus of smoothness and obtained the following result: for any $f \in$ $C_{B}(T)$,

$$
\left\|V_{n, d} f-f\right\|_{\infty} \leq C \omega_{\varphi}^{2}\left(f, \frac{1}{\sqrt{n}}\right)_{\infty},
$$

where

$$
\begin{gathered}
\omega_{\varphi}^{r}(f, t)_{\infty}=\sup _{0 \leq h \leq t} \sum_{i=1}^{d}\left\|\Delta_{h \varphi_{i} \mathbf{e}_{i}}^{r} f\right\|_{\infty}, \\
\Delta_{h \mathbf{e}}^{r} f(\mathbf{x})= \begin{cases}0, & \text { else, } \\
\sum_{i=0}^{r}\left(\begin{array}{l}
r \\
i
\end{array}\right)(-1)^{i} f(\mathbf{x}+i h \mathbf{e}), & \mathbf{x}, \mathbf{x}+r h \mathbf{e} \in T .\end{cases}
\end{gathered}
$$

The $r$ th $K$-functional is defined as follows

$$
\begin{aligned}
& K_{\varphi}^{r}(f ; t) \\
& \quad=\inf _{g}\left\{\|f-g\|_{\infty}+t^{r} \sum_{i=1}^{d}\left(\left\|\varphi_{i}^{r} D_{i}^{r} g\right\|_{\infty}\right), g \in C_{\varphi}^{2}(T)\right\} .
\end{aligned}
$$

And the $r$ th modulus of smoothness is

$$
\omega_{\varphi}^{r}(f, t)_{\infty}=\sup _{0 \leq h \leq t} \sum_{i=1}^{d}\left\|\Delta_{h \varphi_{i} \mathbf{e}_{i}}^{r} f\right\|_{\infty} .
$$

Naturally, we will consider the following problem: "are there similar results in the approximation with weights by multivariate Baskakov operator?" However, approximation with Jacobi weights $\left(\omega(\mathbf{x})=\mathbf{x}^{\alpha}(1+|\mathbf{x}|)^{-b}(0<|\alpha|<1, b>\right.$ $0))$ is not a simple generalization of normal approximation means. In the norm $\|\omega f\|_{\infty}$, the multivariate Baskakov operator is not bounded. By introducing the weighted norm

$$
\|f\|_{\omega}=\|\omega f\|_{\infty}+f(\mathbf{0}),
$$

we find that the multivariate Baskakov operator is bounded, and thus we can investigate approximation capability of the operators. In the previous weighted norm, the $r$ th weighted $K$-functional is defined as follows:

$$
\begin{array}{r}
K_{\varphi}^{r}(f ; t)_{\omega} \\
=\inf _{g}\left\{\|f-g\|_{w}+t^{r} \sum_{i=1}^{d}\left(\left\|\varphi_{i}^{r} D_{i}^{r} g\right\|_{\omega}\right), g \in C_{\varphi}^{2}(T)\right\}, \\
f \in C_{B}(T),
\end{array}
$$

and $r$ th weighted Ditzian-Totik modulus of smoothness is

$$
\omega_{\varphi}^{r}(f, t)_{\omega}=\sup _{0 \leq h \leq t} \sum_{i=1}^{d}\left\|\Delta_{h \varphi_{i} \mathbf{e}_{i}}^{r} f\right\|_{\omega}, \quad g \in C_{B}(T),
$$

where $\varphi_{i}^{2}(\mathbf{x})=x_{i}(1+|\mathbf{x}|)$
Using the previous notations, we will present our results. Firstly, we study the unboundedness of multivariate Baskakov operator in the normal norm.

Theorem 1. Let $f \in C_{B}(T)$; then the operator $V_{n, d}(f ; x)$ is unbounded in the norm $\|\omega f\|_{\infty}$.

In addition, introducing the new norm, we establish the upper bound estimation of weighted approximation by multivariate Baskakov operator.

Theorem 2. Let $f \in C_{B}(T)$; then one has

$$
\left\|V_{n, d} f-f\right\|_{\omega} \leq C \omega_{\varphi}^{2}\left(f, \frac{1}{\sqrt{n}}\right)_{\omega}
$$

Remark 3. Our result reveals two things: (i) for any multivariate bounded continuous function $f \in C_{B}(T)$, there is a multivariate polynomial $V_{n, d}(f)$ that approximates $f$ arbitrarily well (when $n$ is sufficiently large) in the continuous space. (ii) Quantitatively, the approximation accuracy of a polynomial $V_{n, d}(f)$ can be controlled by the $\omega_{\varphi}^{2}(f, 1 / \sqrt{n})_{\omega}$; here $\omega_{\varphi}^{2}(f, 1 / \sqrt{n})_{\omega}$ is the weighted smoothness modulus of the function $f$.

\section{Some Lemmas}

In order to prove our results, we will show some lemmas in this section.

Lemma 4 (see [6]). For $f \in C_{\omega}, 0 \leq \lambda \leq 1$, one has

$$
\left\|V_{n, 1} f-f\right\|_{\omega} \leq C \omega_{\varphi}^{2}\left(f, \frac{1}{\sqrt{n}}\right)_{\omega}
$$

Lemma 5 (see [5]). For $c, d \geq 0$,

$$
\left|\sum_{k=1}^{\infty}\left(\frac{k}{n}\right)^{-c}\left(1+\frac{k}{n}\right)^{d} v_{n, k}(x)\right| \leq C x^{-c}(1+x)^{d}, \quad x>0 .
$$

Lemma 6. For $f \in C_{B}(T)$, then one has

$$
\left|\omega(\mathbf{x}) \sum_{\mathbf{k}=1}^{\infty} v_{n, \mathbf{k}}(\mathbf{x}) f\left(\frac{\mathbf{k}}{n}\right)\right| \leq C\|\omega f\|_{\infty} .
$$


Proof. For convenience, we only prove the case of $d=2$; for a general $d$, we can prove similarly. For $d=2$, we have

$$
\begin{aligned}
& \left|\omega(\mathbf{x}) \sum_{\mathbf{k}=1}^{\infty} v_{n, \mathbf{k}}(\mathbf{x}) f\left(\frac{\mathbf{k}}{n}\right)\right| \\
& =\left|\omega(\mathbf{x}) \sum_{k_{1}=1}^{\infty} v_{n, k_{1}}\left(x_{1}\right) \sum_{k_{2}=1}^{\infty} v_{n+k_{1}, k_{2}}\left(\frac{x_{2}}{1+x_{1}}\right) f\left(\frac{k_{1}}{n}, \frac{k_{2}}{n}\right)\right| \\
& =\mid \omega(\mathbf{x}) \sum_{k_{1}=1}^{\infty} v_{n, k_{1}}\left(x_{1}\right) \sum_{k_{2}=1}^{\infty} v_{n+k_{1}, k_{2}}\left(\frac{x_{2}}{1+x_{1}}\right) f\left(\frac{k_{1}}{n}, \frac{k_{2}}{n}\right) \\
& \times \omega\left(\frac{k_{1}}{n}, \frac{k_{2}}{n}\right)\left(\frac{k_{1}}{n}\right)^{-\alpha_{1}}\left(\frac{k_{2}}{n}\right)^{-\alpha_{2}} \times\left(1+\frac{k_{1}}{n}+\frac{k_{2}}{n}\right)^{-b} \\
& \leq C\|\omega f\|_{\infty} \mid \omega(\mathbf{x})\left\{\sum_{k_{1}=1}^{\infty} v_{n, k_{1}}\left(x_{1}\right)\left(\frac{k_{1}}{n}\right)^{-\alpha_{1}}\right\} \\
& \times\left\{\sum_{k_{2}=1}^{\infty} v_{n+k_{1}, k_{2}}\left(\frac{x_{2}}{1+x_{1}}\right)\left(\frac{k_{2}}{n}\right)^{-\alpha_{2}}\right. \\
& \left.\times\left(1+\frac{k_{1}}{n}+\frac{k_{2}}{n}\right)^{-b}\right\} \\
& =C\|\omega f\|_{\infty} \mid \omega(\mathbf{x})\left\{\sum_{k_{1}=1}^{\infty} v_{n, k_{1}}\left(x_{1}\right)\left(\frac{k_{1}}{n}\right)^{-\alpha_{1}}\right\} \\
& \times\left\{\sum_{k_{2}=1}^{\infty} v_{n+k_{1}, k_{2}}\left(\frac{x_{2}}{1+x_{1}}\right)\left(1+\frac{k_{2}}{n+k_{1}}\right)^{b}\right. \\
& \left.\times\left(\frac{k_{2}}{n+k_{1}}\right)^{-\alpha_{2}}\right\} \\
& \leq C\|\omega f\|_{\infty} \mid \omega(\mathbf{x}) x_{1}^{-\alpha_{1}} x_{2}^{-\alpha_{2}}\left(1+x_{1}+x_{2}\right)^{b} \\
& \leq C\|\omega f\|_{\infty} \text {. }
\end{aligned}
$$

In the previous derivation, Lemma 5 and the following inequality were used:

$$
\sum_{k=0}^{\infty} v_{n, k}(x)\left(\frac{n}{k+1}\right)^{l} \leq C x^{-l}, \quad l \in N .
$$

\section{The Proof of Theorems}

In this section, we will show the unboundedness of multivariate Baskakov operator in the general weighted norm and then give the proof of Theorem 2 .
Proof of Theorem 1. Let

$$
f_{m}\left(x_{1}, x_{2}\right)=\frac{1}{x_{1}^{\alpha_{1}} x_{2}^{\alpha_{2}}+(1 / m)} .
$$

Then we have

$$
\begin{aligned}
\left\|\omega f_{m}\right\|_{\infty} & =\max _{x>0}\left|x_{1}^{-\alpha_{1}} x_{2}^{-\alpha_{2}}\left(1+x_{1}+x_{2}\right)^{-b} \frac{1}{x_{1}^{\alpha_{1}} x_{2}^{\alpha_{2}}+(1 / m)}\right| \\
& \leq\left(\frac{1}{1+x_{1}+x_{2}}\right)^{b} \leq 1 .
\end{aligned}
$$

On the other hand,

$$
\begin{aligned}
& \left\|\omega V_{n, d} f\right\|_{\infty} \\
& =\max _{x>0}\left|\omega(\mathbf{x}) \sum_{k=0}^{\infty} V_{n, k}(x) f\left(\frac{k}{n}\right)\right| \\
& \geq \max _{x>0}\left|\omega(\mathbf{x}) f_{m}(0,0) v_{n, 0}(x)\right| \\
& \quad-\max _{x>0}\left|\omega(\mathbf{x}) \sum_{k=1}^{\infty} v_{n, k}(x) f\left(\frac{k}{n}\right)\right| \\
& \geq \max _{x>0}\left|\omega(\mathbf{x}) f_{m}(0,0)\left(1+x_{1}+x_{2}\right)^{-n}\right|-C \\
& \geq m\left(\frac{3}{2}\right)^{n+b}-C \longrightarrow \infty(m \longrightarrow \infty) .
\end{aligned}
$$

As we know, multivariate Baskakov operator $V_{n, d} f$ has the property of preserve linear, for convenience the following discussion, we may suppose $f(\mathbf{0})=0$.

Proof of Theorem 2. In order to prove Theorem 2, we first show the following inequality:

$$
\left\|V_{n, d}(f)-f\right\|_{\omega} \leq \begin{cases}\|f\|_{\omega}, & f \in C_{B}(T), \\ n^{-1} \sum_{i=0}^{d}\left\|\varphi_{i}^{2} D_{i}^{2} f\right\|_{\omega}, & f \in C_{\varphi}^{2}(T) .\end{cases}
$$

For the first inequality, it is obviously correct since $\left\|V_{n, d} f\right\|_{\omega} \leq C\|f\|_{\omega}$. In the following we will certify the second inequality by the mathematical induction. Our proof is based on an induction argument for the dimension $d$. We will also use a method called decomposition technique for the multivariate Baskakov operator.

(i) For $d=1$, from Lemma 4, we have

$$
\left\|V_{n, d}(f)-f\right\|_{\omega} \leq n^{-1}\left\|\varphi^{2} f^{\prime \prime}\right\|_{\omega} .
$$

(ii) When $d=r$, suppose that

$$
\left\|V_{n, d}(f)-f\right\|_{\omega} \leq\left\|\varphi_{i}^{2} D_{i}^{2} f\right\|_{\omega}
$$


4

Journal of Function Spaces and Applications

is established. So when $d=r+1$, using the multivariate decomposition technique in [7], we have

$$
V_{n, d}(f, x)=\sum_{k_{1}=0}^{\infty} v_{n, k_{1}}(x) \sum_{k^{*}=0}^{\infty} v_{n+k_{1}, \mathbf{k}^{*}}\left(\frac{\mathbf{x}^{*}}{1+x_{1}}\right)
$$

where

$$
\begin{array}{ll}
\mathbf{x}^{*}=\left(x_{2}, x_{3}, \ldots, x_{n}\right), & \mathbf{x}=\left(x_{1}, \mathbf{x}^{*}\right), \\
\mathbf{k}^{*}=\left(k_{2}, k_{3}, \ldots, k_{d}\right), & \mathbf{k}=\left(k_{1}, \mathbf{k}^{*}\right) .
\end{array}
$$

Let

$$
\begin{aligned}
& g_{k_{1}}(\mathbf{u})=f\left(\frac{k_{1}}{n},\left(1+\frac{k_{1}}{n}\right) \mathbf{u}\right), \quad \mathbf{u} \in T_{d-1}, \\
& h(t)=h(t, \mathbf{x})=f\left(t,(1+t) \frac{\mathbf{x}^{*}}{1+x_{1}}\right) .
\end{aligned}
$$

We get

$$
\begin{aligned}
\omega(\mathbf{x}) & \left(V_{n, d}(f, \mathbf{x})\right)-f(\mathbf{x}) \\
= & \omega(\mathbf{x})\left(\sum_{k_{1}=0}^{\infty} v_{n, k_{1}}\left(x_{1}\right)\left(V_{n+k_{1}, d-1}\left(g_{k_{1}}(\cdot), \mathbf{z}\right)-g_{k_{1}}(\mathbf{z})\right)\right) \\
& +\left(V_{n, 1}\left(h(\cdot), x_{1}\right)-h\left(x_{1}\right)\right) \\
= & P+Q
\end{aligned}
$$

where $z=\mathbf{x}^{*} /\left(1+x_{1}\right)$, and we have

$$
\begin{aligned}
|P| & =\left|\omega(\mathbf{x})\left(\sum_{k_{1}=0}^{\infty} v_{n, k_{1}}\left(x_{1}\right)\left(V_{n+k_{1}, d-1}\left(g_{k_{1}}(\cdot), \mathbf{z}\right)-g_{k_{1}}(\mathbf{z})\right)\right)\right| \\
= & \mid x_{1}^{\alpha_{1}}\left(1+x_{1}\right)^{-b+\left|\alpha^{*}\right|} \sum_{k_{1}=0}^{\infty} v_{n, k_{1}}\left(x_{1}\right) \omega(\mathbf{z}) \\
& \times\left(V_{n+k_{1}, d-1}\left(g_{k_{1}}(\cdot), \mathbf{z}\right)-g_{k_{1}}(\mathbf{z})\right) \mid \\
\leq & x_{1}^{\alpha_{1}}\left(1+x_{1}\right)^{-b+\left|\alpha^{*}\right|} \sum_{k_{1}=0}^{\infty} \frac{1}{n+k_{1}} v_{n, k_{1}}\left(x_{1}\right) \sum_{i=1}^{r}\left\|\varphi_{i}^{2} D_{i}^{2} f\right\|_{\omega}
\end{aligned}
$$

The previous derivation used the following inequality:

$$
\begin{aligned}
\omega(\mathbf{x}) & =\mathbf{x}^{\alpha}(1+|\mathbf{x}|)^{-b} \\
= & x_{1}^{\alpha_{1}}\left(\frac{x^{*}}{1+x_{1}}\right)^{\alpha^{*}}\left(1+x_{1}\right)^{-b+\left|\alpha^{*}\right|}\left(1+\frac{x^{*}}{1+x_{1}}\right)^{-b} \\
= & x_{1}^{\alpha_{1}}\left(1+x_{1}\right)^{-b+\left|\alpha^{*}\right|} \omega(\mathbf{z}), \\
\varphi_{i}^{2}(\mathbf{u}) & D_{i}^{2}(\mathbf{u}) g_{k_{1}}(\mathbf{u}) \omega(\mathbf{u}) \\
= & \mathbf{u}_{i}(1+|\mathbf{u}|)\left(1+\frac{k_{1}}{n}\right) D_{i+1}^{2} f\left(\frac{k_{1}}{n},\left(1+\frac{k_{1}}{n}\right) \mathbf{u}\right) \\
& \times \mathbf{u}^{\alpha^{*}}(1+|\mathbf{u}|)^{-b} \\
= & \left(\frac{n}{k_{1}}\right)^{\alpha_{1}}\left(\frac{n}{n+k_{1}}\right)^{-b+\left|\alpha^{*}\right|} \omega \varphi_{i+1}^{2} D_{i+1}^{2} f\left(\frac{k_{1}}{n},\left(1+\frac{k_{1}}{n}\right) \mathbf{u}\right) .
\end{aligned}
$$

So we get

$$
\left\|\varphi_{i}^{2} D_{i}^{2} g_{k_{1}}\right\|_{\omega} \leq\left(\frac{n}{k_{1}}\right)^{\alpha_{1}}\left(\frac{n}{n+k_{1}}\right)^{-b+\left|\alpha^{*}\right|}\left\|\varphi_{i}^{2} D_{i}^{2} f\right\|_{\omega} .
$$

From Lemma 6, we have

$$
\begin{aligned}
|P| \leq & \left|x_{1}^{\alpha_{1}}\left(1+x_{1}\right)^{-b+\left|\alpha^{*}\right|} \sum_{k_{1}=0}^{\infty} \frac{1}{n+k_{1}} p_{n, k_{1}}\left(x_{1}\right)\right| \\
& \times\left(\frac{n}{k_{1}}\right)^{\alpha_{1}}\left(\frac{n}{n+k_{1}}\right)^{-b+\left|\alpha^{*}\right|}\left\|\varphi_{i}^{2} D_{i}^{2} f\right\|_{\omega} \\
\leq & \frac{C}{n}\left\|\varphi_{i}^{2} D_{i}^{2} f\right\|_{\omega} .
\end{aligned}
$$

In the following, we will establish the estimation of $Q$ :

$$
\begin{aligned}
|Q|= & \left|\left(V_{n, 1}\left(h(\cdot), x_{1}\right)-h\left(x_{1}\right)\right) \omega(\mathbf{x})\right| \\
= & \left|x_{1}^{\alpha_{1}}\left(\mathbf{x}^{*}\right)^{\alpha^{*}}(1+|\mathbf{x}|)^{-b}\left(B_{n, 1}\left(h(\cdot), x_{1}\right)-h\left(x_{1}\right)\right)\right| \\
= & \mid\left(\mathbf{x}^{*}\right)^{\alpha^{*}}\left(\frac{1+|x|}{1+x_{1}}\right)^{-b}\left(1+x_{1}\right)^{-b} x_{1}^{\alpha_{1}} \\
& \times\left(V_{n, 1}\left(h(\cdot), x_{1}\right)-h\left(x_{1}\right)\right) \mid \\
\leq & \frac{C}{n}\left|\left(\mathbf{x}^{*}\right)^{\alpha^{*}}\left(\frac{1+|x|}{1+x_{1}}\right)^{-b}\right|\left\|\varphi^{2} h^{\prime \prime}\right\|_{\omega}
\end{aligned}
$$


and for the term $\left\|\varphi^{2} h^{\prime \prime}\right\|_{\omega}$, we have the following estimation:

$$
\begin{aligned}
& \left\|\varphi^{2} h^{\prime \prime}\right\|_{\omega} \\
& =\max _{0 \leq t<\infty}\left|t(1+t) t^{\alpha_{1}}(1+t)^{-b} h^{\prime \prime}\left(t,(1+t) \frac{\mathbf{x}^{*}}{1+x_{1}}\right)\right| \\
& =\max _{0 \leq t<\infty}\left|t(1+t) t^{\alpha_{1}}(1+t)^{-b}\right| D_{1}^{2} f \\
& \quad+\sum_{i=2}^{d} \frac{\mathbf{x}_{i}}{1+x_{1}} D_{1 i}^{2} f+\sum_{i=2}^{d} \frac{\mathbf{x}_{i}}{1+x_{1}} D_{i 1}^{2} f \\
& \quad+\sum_{i=2}^{d} \sum_{j=2}^{d} \frac{x_{i} x_{j}}{\left(1+x_{1}\right)^{2}} D_{i, j}^{2} f\left(t,(1+t) \frac{\mathbf{x}^{*}}{1+x_{1}}\right) \\
& =\max _{0 \leq t<\infty}\left|t(1+t) t^{\alpha_{1}}(1+t)^{-b} L_{1}\right|,
\end{aligned}
$$

where

$$
\begin{aligned}
L_{1}= & \mid \frac{1+x_{1}}{1+|\mathbf{x}|} \varphi_{1}^{2} D_{1}^{2} f+\sum_{i=2}^{d} \varphi_{1 i}^{2} D_{1 i}^{2} f+\sum_{i=2}^{d} \varphi_{i 1}^{2} D_{i 1}^{2} f \\
& +\sum_{i=2}^{d} \frac{t}{1+t} \frac{\mathbf{x}_{i}}{1+|\mathbf{x}|} \varphi_{i}^{2} D_{i}^{2} f \\
& +\sum_{i, j=2, i \neq j}^{d} \varphi_{i j}^{2} D_{i j}^{2} f\left(t,(1+t) \frac{\mathbf{x}^{*}}{1+x_{1}}\right) \mid .
\end{aligned}
$$

Note that

$$
\begin{aligned}
& \omega\left(t,(1+t) \frac{\mathbf{x}^{*}}{1+x_{1}}\right) \\
& \quad=\left(\mathbf{x}^{*}\right)^{\alpha^{*}}\left(\frac{1+t}{1+x_{1}}\right)^{\left|\alpha^{*}\right|} t^{\alpha_{1}}(1+t)^{-b}\left(\frac{1+|\mathbf{x}|}{1+x_{1}}\right)^{-b}
\end{aligned}
$$

We have

$$
\left\|\varphi^{2} h^{\prime \prime}\right\|_{\omega}=\left(\mathbf{x}^{*}\right)^{-\alpha^{*}}\left(\frac{1+|x|}{1+x_{1}}\right)^{b}\left(\frac{1+t}{1+x_{1}}\right)^{-\left|\alpha^{*}\right|} L_{2} .
$$

Here

$$
\begin{aligned}
\left|L_{2}\right|= & \mid \frac{1+x_{1}}{1+|\mathbf{x}|} \omega \varphi_{1}^{2} D_{1}^{2} f+\sum_{i=2}^{d} \omega \varphi_{1 i}^{2} D_{1 i}^{2} f+\sum_{i=2}^{d} \omega \varphi_{i 1}^{2} D_{i 1}^{2} f \\
& +\sum_{i=2}^{d} \frac{t}{1+t} \frac{\mathbf{x}_{i}}{1+|\mathbf{x}|} \omega \varphi_{i}^{2} D_{i}^{2} f \\
& +\sum_{i, j=2, i \neq j}^{d} \omega \varphi_{i j}^{2} D_{i j}^{2} f\left(t,(1+t) \frac{\mathbf{x}^{*}}{1+x_{1}}\right) \mid .
\end{aligned}
$$

From [7], we have

$$
\begin{gathered}
\left|D_{i, j}^{2} f(\mathbf{x})\right| \leq \sup _{1 \leq i \leq d}\left|D_{i}^{2} f(\mathbf{x})\right|, \\
\varphi_{i, j}(\mathbf{x}) \leq \min \left\{\varphi_{i}(\mathbf{x}), \varphi_{j}(\mathbf{x})\right\}, \\
\left\|\varphi^{2} h^{\prime \prime}\right\|_{\omega}=\left(\mathbf{x}^{*}\right)^{-\alpha^{*}}\left(\frac{1+|x|}{1+x_{1}}\right)^{b}\left(\frac{1+t}{1+x_{1}}\right)^{-\left|\alpha^{*}\right|}\left\|\varphi_{i}^{2} D_{i}^{2} f\right\|_{\omega} .
\end{gathered}
$$

Hence

$$
|Q| \leq \frac{C}{n}\left\|\varphi_{i}^{2} D_{i}^{2} f\right\|_{\omega}
$$

Thus we have

$$
\begin{aligned}
& \left\|V_{n, d} f-f\right\|_{\omega} \\
& \quad=\left\|V_{n, d} f-V_{n, d} g+V_{n, d} g-g+g-f\right\|_{\omega} \\
& \leq C\left\|V_{n, d} f-V_{n, d} g\right\|_{\omega}+\|f-g\|_{\omega} \\
& \quad \leq \frac{C}{n}\left\|\varphi_{i}^{2} D_{i}^{2} f\right\|_{\omega}+\|f-g\|_{\omega} \\
& \leq C K_{r}^{2}\left(f, \frac{1}{\sqrt{n}}\right)_{\omega} \\
& \quad \leq C \omega_{\varphi}^{2}\left(f, \frac{1}{\sqrt{n}}\right)_{\omega} .
\end{aligned}
$$

Here we used the following facts: for $f \in C_{B}(T)$, then

$$
\frac{1}{C} \omega_{\varphi}^{r}(f, t)_{\omega} \leq K_{\varphi}^{r}(f ; t)_{\omega} \leq C \omega_{\varphi}^{r}(f, t)_{\omega} .
$$

The result is easily obtained from [7-9]. Theorem 2 is completed.

\section{References}

[1] V. A. Baskakov, "An instance of a sequence of linear positive operators in the space of continuous functions," Doklady Akademii Nauk SSSR, vol. 113, no. 2, pp. 249-251, 1957.

[2] Z. Ditzian, "On global inverse theorems of Szász and Baskakov operators," Canadian Journal of Mathematics, vol. 31, no. 2, pp. 255-263, 1979.

[3] M. Becker, "Global approximation theorems for Szász-Mirakjan and Baskakov operators in polynomial weight spaces," Indiana University Mathematics Journal, vol. 27, no. 1, pp. 127-142, 1978.

[4] J. J. Wang, The weighted approximation of some linear operators [M.S. thesis], Ningxia University, 2003.

[5] P. C. Xuan and D. X. Zhou, "The order of convergence for weighted Baskakov operators," Acta Mathematicae Applicatae Sinica, vol. 18, no. 1, pp. 129-139, 1995.

[6] J. J. Wang and Y. C. Xue, "Direct and inverse approximation theorems with Jacobi weight for Baskakov operators and their derivatives," Chinese Annals of Mathematics A, vol. 26, no. 4, pp. 561-570, 2005.

[7] F. Cao, C. Ding, and Z. Xu, "On multivariate Baskakov operator," Journal of Mathematical Analysis and Applications, vol. 307, no. 1, pp. 274-291, 2005. 
[8] Z. Ditzian and V. Totik, Moduli of Smoothness, Springer, New York, NY, USA, 1987.

[9] F. L. Cao and X. D. Zhang, "Degree of convergence with Jacobi weight for $d$-dimensional Bernstein operators," Mathematica Numerica Sinica, vol. 23, no. 4, pp. 407-416, 2001. 


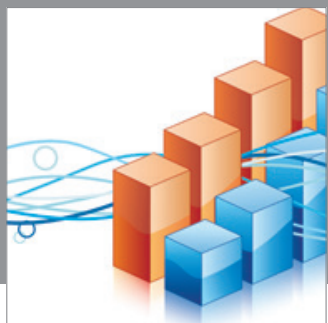

Advances in

Operations Research

mansans

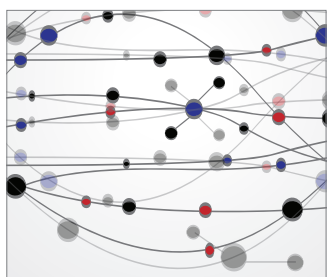

The Scientific World Journal

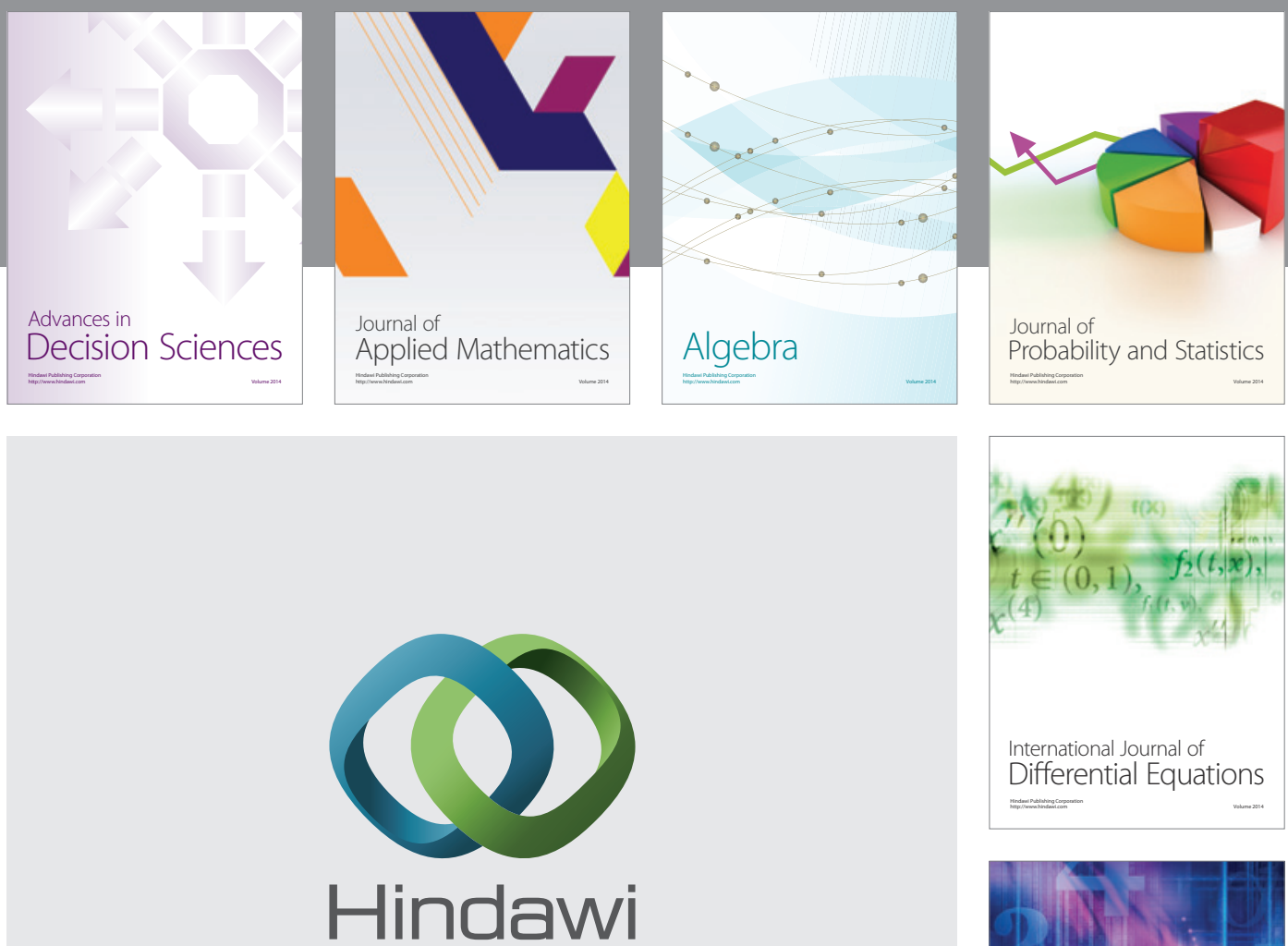

Submit your manuscripts at http://www.hindawi.com
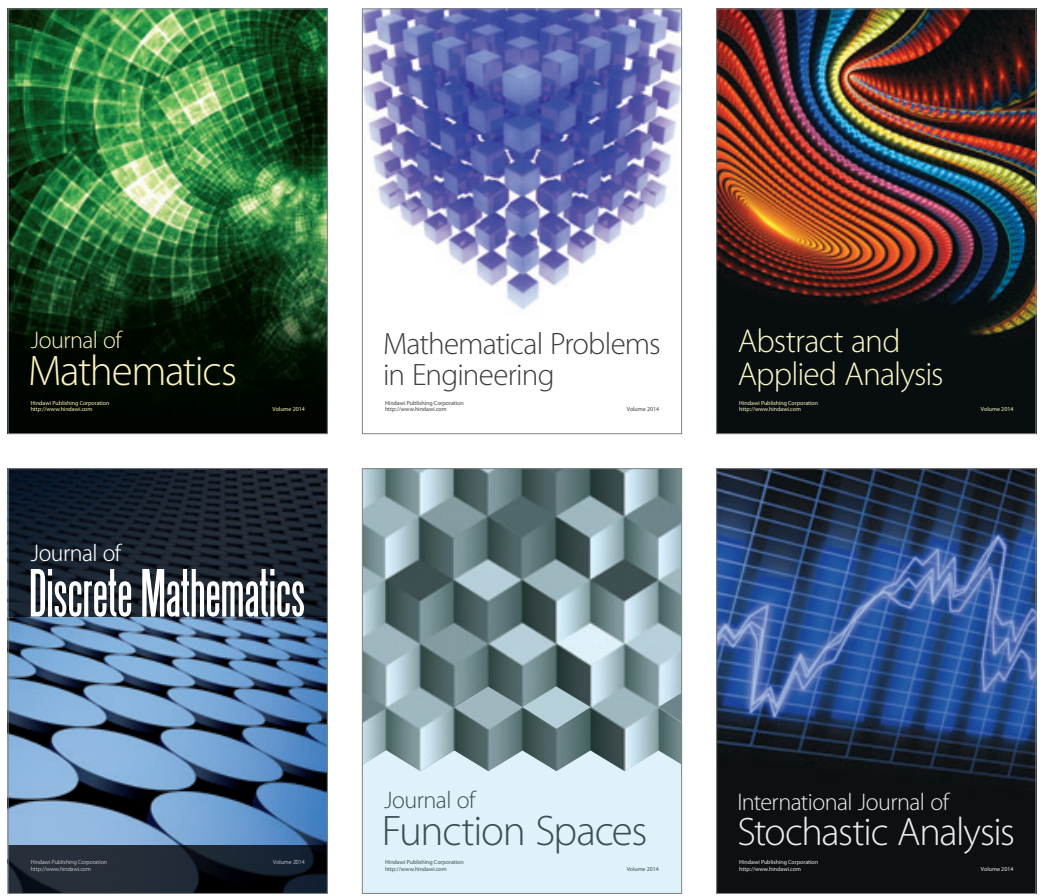

Journal of

Function Spaces



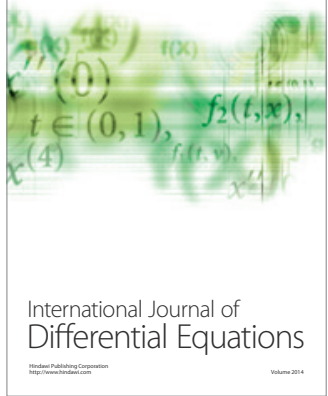
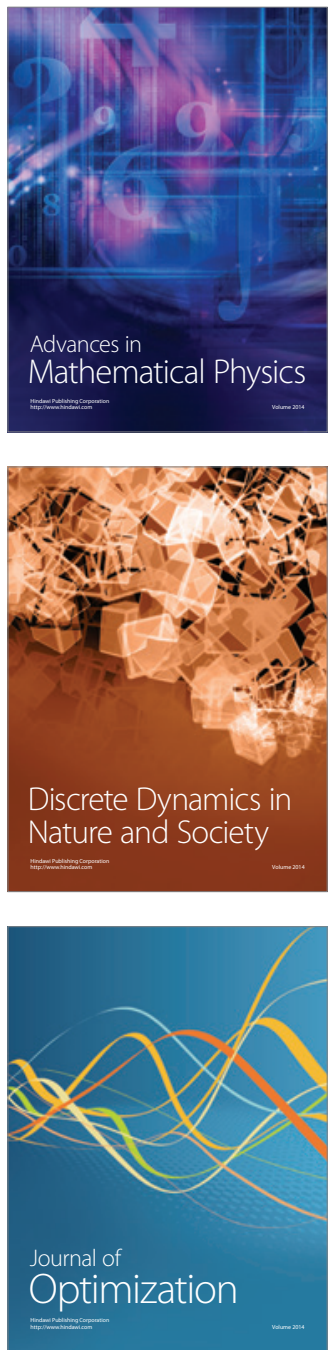